\title{
Accretion disc variability in AGN
}

\author{
Patricia Arévalo \\ Universidad Andres Bello, Av. Republica 252, Santiago, Chile \\ email: parevalo@unab.cl
}

\begin{abstract}
The central engine of AGN is too compact to be observed directly so its structure has to be inferred through the combination of spectral and variability information. Here we study the connection between optical-emitting accretion disc and X-ray emitting corona of the Seyfert NGC3783. Short light curves are consistent with optical fluctuations arising from X-ray reprocessing, but the long-term fluctuations found over the course of several years prove that at least long-term optical variability is not produced by the X-rays: at time-scales longer than 1 year the variability power in the optical is higher than in the X-rays. This indicates variability originating in the thin accretion disc itself, therefore capable of revealing its characteristics.
\end{abstract}

Keywords. Galaxies: active, galaxies: Seyfert, X-rays: galaxies

\section{Introduction}

Standard accretion disc theory (Shakura \& Sunyaev, 1973) limits the range of accretion disc emission to the optical or UV, while the observed X-rays are expected to be be produced by Compton up-scattering of thermal photons by a corona, whose location, geometry and behaviour are unclear. Time delays between similar changes in optical and X-ray flux in short light curves indicate that the optical variability arises from reprocessing of variable X-rays by the optically thick accretion disc. If this were true at all time-scales it would be impossible to study the structure of the disc itself through variability. Long term, well sampled light curves taken simultaneously in optical and X-ray bands show that the true scenario is more complex (Arévalo et al. 2009).

\section{Disc and corona variability}

The X-ray power spectrum is well described by a broken power law with a break timescale $T_{b}$ around $10^{5} \mathrm{~s}$ or 1 day. $T_{b}$ has been recognised as the only characteristic time-scale of coronal variability in almost all AGN studied (McHardy et al. 2006) and must be related to the fastest processes happening in the corona, probably the viscous time-scale of a geometrically thick accretion flow at its innermost region. The optical power spectrum on the other hand, resembles an unbroken powerlaw down to the longest time-scales probed, surpassing the X-ray power at timescales of $\sim 300$ days.

The fluctuations on different time-scales can be separated by convolving them with a Mexican hat profile, which effectively removes fluctuations on longer and shorter timescales than the filter width, while keeping the calculation in time-domain prevents problems produced by irregularly-sampled light curves (Arévalo et al. 2012).

The plots in Fig. 1 show the observed light curves filtered on the timescales displayed on each panel. On year-long time-scales the optical band flux changes with larger amplitude than the X-rays and the fluctuations are not clearly correlated at 0 time lag. These optical fluctuations must be intrinsic to the disc. More rapid variability shows good correspondence between optical and X-ray bands, the amplitudes are similar and the fluctuations well correlated. These fluctuations are delayed by no more than a few days 

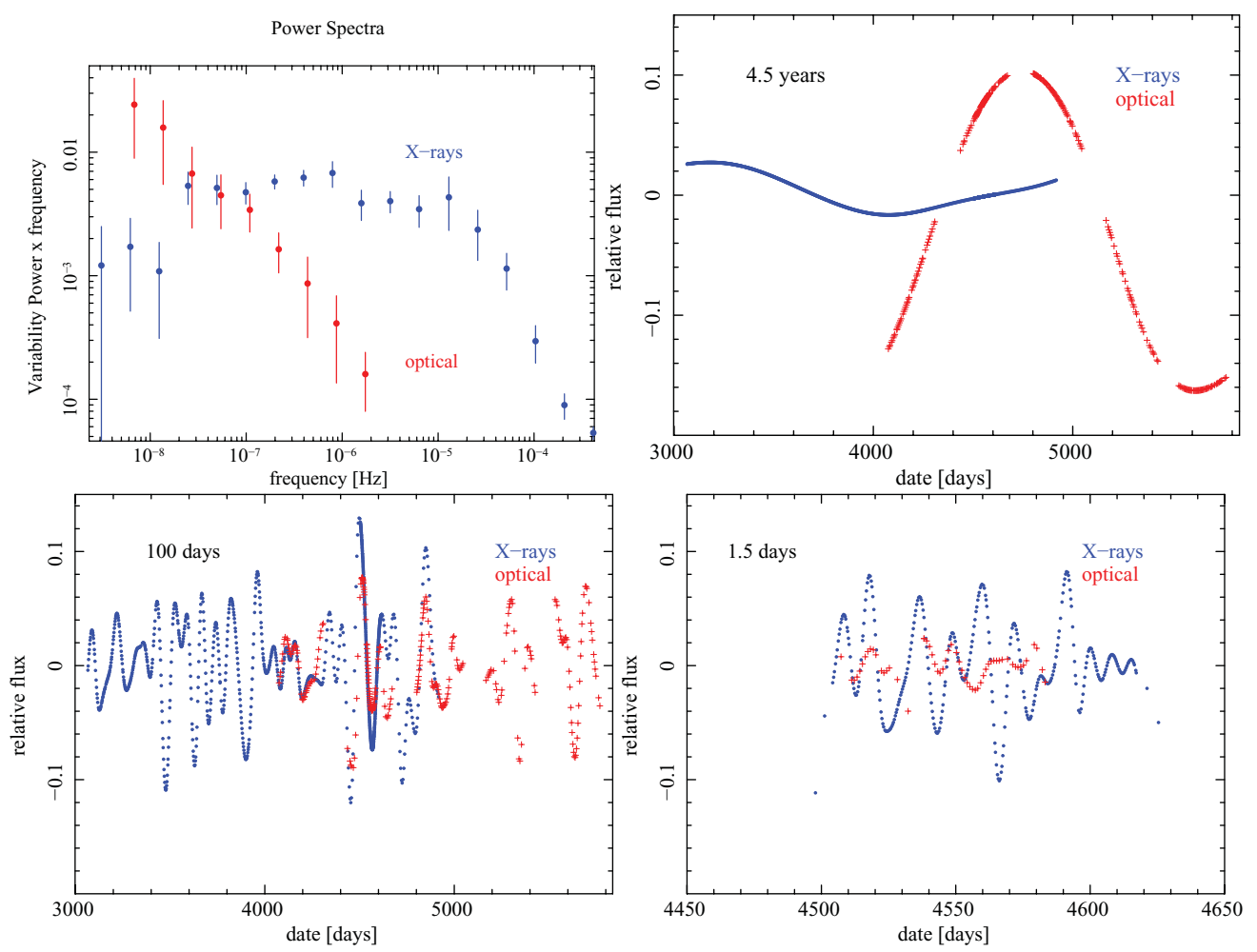

Figure 1. Top right: power spectra of optical (single power law) and X-ray (broken powerlaw) fluctuations. Rest: X-ray and optical lightcurves filtered on the timescales noted on each panel.

with respect to each other, so the emitting regions must be closely connected. Short timescale fluctuations display the well known behaviour of higher amplitude flux variations in the X-rays. These might be due to reprocessing of X-rays by the accretion disc, damped by light travel time to different edges of the reprocessor.

\section{Conclusions}

The comparison of optical and X-ray power spectra shows that: Long term optical variability must be intrinsic to the disc. Reprocessing of X-rays cannot be a major contributor to disc variability on time-scales longer than 300 days in NGC3783. The disc power spectrum is a power law of slope -2 down to the longest time-scales probed. The time-scale where the power spectrum breaks must be similar to or longer than the length of the light curve, i.e. 5 years. Therefore the difference in characteristic time-scales in disc and corona is at least a factor of 1000 . If both regions are accretion flows fluctuating on their own viscous time-scale (Churazov et al. 2001), we can conclude that the product of their structural parameters $\alpha(H / r)^{2}$ differs at least by this ratio of 1000 .

\section{References}

Shakura \& Sunyaev 1973, A\&A A, 24, 377

Churazov, Gilfanov, Revnivtsev 2001, MNRAS, 321,759

Arévalo, et al. 2009 MNRAS, 397, 2004

McHardy, et al. 2006, Nature, 444, 730

Arévalo, et al. 2012, MNRAS, 426, 1793 